\title{
Novel Predictor of Lung Injury After Balloon Pulmonary Angioplasty in Patients With Chronic Thromboembolic Pulmonary Hypertension
}

\author{
Satoshi AKAGI, ${ }^{1}$ MD
}

$\mathrm{C}$ hronic thromboembolic pulmonary hypertension (CTEPH) is a form of pulmonary hypertension classified as group IV. ${ }^{1)}$ Pulmonary artery stenosis or obstruction by an organized thrombus can cause pulmonary hypertension, which can lead to right heart failure and death. Medical therapy, balloon pulmonary angioplasty (BPA), and pulmonary endarterectomy are recommended for the treatment of CTEPH. In recent guidelines, pulmonary endarterectomy is recommended for patients with CTEPH who are technically operable. Medical therapy or BPA is recommended for patients with CTEPH who are technically non-operable. ${ }^{1)}$

The prognosis of patients with CTEPH depends on the degree of mean pulmonary artery pressure (PAP). ${ }^{2}$ The prognosis of patients with CTEPH whose mean PAP is over 30 $\mathrm{mmHg}$ is very poor. In other words, sufficient reduction of mean PAP by medical treatment has the potential to improve the prognosis of patients with CTEPH. Riociguat, which is a soluble guanylate cyclase stimulator, is currently available for treating CTEPH. Although riociguat has been shown to improve exercise capacity and pulmonary vascular resistance, it could not induce sufficient reduction of mean PAP. ${ }^{3)}$ On the other hand, BPA results in a remarkable reduction of mean PAP and is expected to improve the prognosis of patients with $\mathrm{CTEPH}{ }^{4)}$ Therefore, BPA should be selected for patients with CTEPH who are technically non-operable.

The first full report on BPA in patients with CTEPH was published in 2001. 5) Although it was shown that BPA improved exercise capacity and mean PAP, reperfusion pulmonary edema occurred at a high rate and the 30-day mortality rate was $5.5 \%$. Therefore, BPA was abandoned for several years. Matsubara, et al performed BPA to reduce reperfusion pulmonary edema after BPA through a trial and error process. The use of epoprostenol to decrease mean PAP before BPA and the use of methylprednisolone and non-invasive positive-pressure ventilation after BPA could not prevent the occurrence of reperfusion pulmonary edema. From their experience with BPA, they noticed that the lung complication after BPA was not reperfusion pulmonary edema but rather vascular injury caused by a catheter-related technique. They therefore inserted the guide wire carefully and dilated an undersized balloon on the target lesion. This refined BPA technique remarkably decreased mean PAP and exercise capacity with a low rate of lung injury after BPA, ${ }^{4)}$ and their technique is now used worldwide. However, it has not been possible to completely prevent lung injury after BPA. If the factors associated with lung injury after BPA are known, the incidence of lung injury could be decreased by addressing the factors before BPA.

\section{Article p.584}

The pulmonary edema predictive scoring index and mean PAP $>35 \mathrm{mmHg}$ before BPA are useful predictors for lung injury after BPA. ${ }^{5,6)}$ A recent study showed that lung injury after BPA is dependent on the characteristics of the target lesion in CTEPH. ${ }^{7)}$ However, these factors can only be known by using a catheter, which is a very invasive procedure. In this issue of International Heart Journal, Sugimoto, et al reported a novel predictor for lung injury after BPA. ${ }^{8)}$ Their study showed that measurement of pulmonary artery diameter on $\mathrm{CT}$ is useful for the prediction of lung injury after BPA in patients with CTEPH. We could ascertain the risk of lung injury after BPA non-invasively simply by using CT. We should perform BPA more carefully or discuss the indication for BPA if the pulmonary artery diameter index is high.

The study by Sugimoto, et al has several limitations. ${ }^{8)}$ Their study is an observational, single institution study, and the number of subjects was small. In their study, the operator, morphology of the target lesion, and guide wires were not related to lung injury after BPA. However, lung injury after BPA is caused by a catheter-related technique. Therefore, the skill of the operator is very important to examine the factors related to the catheter technique. Further investigation with a large number of subjects, skilled operators, and multiple institutions are required to determine the usefulness of the pulmonary artery diameter index on CT.

\section{REFERENCES}

1. Galiè N, Humbert M, Vachiery JL, et al. 2015 ESC/ERS Guidelines for the diagnosis and treatment of pulmonary hypertension: The Joint Task Force for the Diagnosis and Treatment of Pulmonary Hypertension of the European Society of Cardiology (ESC)

From the ${ }^{1}$ Department of Cardiovascular Medicine, Okayama University Graduate School of Medicine, Dentistry and Pharmaceutical Sciences, Okayama, Japan.

Address for correspondence: Satoshi Akagi, MD, Department of Cardiovascular Medicine, Okayama University Graduate School of Medicine, Dentistry and Pharmaceutical Sciences, 2-5-1 Shikata-cho, Kita-ku, Okayama, Okayama 700-8558, Japan. E-mail: akagi-s@cc.okayama-u.ac.jp

Received for publication May 22, 2017. Accepted May 22, 2017.

Released in advance online on J-STAGE July 14, 2017.

All rights reserved by the International Heart Journal Association. 
and the European Respiratory Society (ERS): Endorsed by: Association for European Paediatric and Congenital Cardiology (AEPC), International Society for Heart and Lung Transplantation (ISHLT). Eur Heart J 2016; 37: 67-119.

2. Riedel M, Stanek V, Widimsky J, Prerovsky I. Longterm followup of patients with pulmonary thromboembolism. Late prognosis and evolution of hemodynamic and respiratory data. Chest 1982; 81: 151-8.

3. Ghofrani HA, D'Armini AM, Grimminger F, et al; CHEST-1 Study Group. Riociguat for the treatment of chronic thromboembolic pulmonary hypertension. N Engl J Med 2013; 369: 319-29.

4. Mizoguchi H, Ogawa A, Munemasa M, Mikouchi H, Ito H, Matsubara $\mathrm{H}$. Refined balloon pulmonary angioplasty for inoperable patients with chronic thromboembolic pulmonary hypertension. Circ Cardiovasc Interv 2012; 5: 748-55.

5. Feinstein JA, Goldhaber SZ, Lock JE, Ferndandes SM, Landzberg MJ. Balloon pulmonary angioplasty for treatment of chronic thromboembolic pulmonary hypertension. Circulation 2001; 103 : 10-3.

6. Inami T, Kataoka M, Shimura N, et al. Pulmonary edema predictive scoring index (pepsi), a new index to predict risk of reperfusion pulmonary edema and improvement of hemodynamics in percutaneous transluminal pulmonary angioplasty. JACC Cardiovasc Interv 2013; 6: 725-36.

7. Kawakami T, Ogawa A, Miyaji K, et al. Novel angiographic classification of each vascular lesion in chronic thromboembolic pulmonary hypertension based on selective angiogram and results of balloon pulmonary angioplasty. Circ Cardiovasc Interv 2016; 9: e003318.

8. Sugimoto K, Nakazato K, Sakamoto N, et al. Pulmonary artery diameter predicts lung injury after nalloon pulmonary angioplasty in patients with chronic thromboembolic pulmonary hypertension. Int Heart J 2017; 58: 584-8. 